\title{
Genome-wide discovery and validation of Eucalyptus small RNAs reveals variable patterns of conservation and diversity across species of Myrtaceae
}

\author{
Marília de Castro Rodrigues Pappas ${ }^{1 *}$, Georgios Joannis Pappas $\mathrm{Jr}^{2}$ and Dario Grattapaglia ${ }^{1,3}$
}

\begin{abstract}
Background: Micro RNAs are a class of small non coding RNAs of 20-24 nucleotides transcribed as single stranded precursors from MIR gene loci. Initially described as post-transcriptional regulators involved in development, two decades ago, miRNAs have been proven to regulate a wide range of processes in plants such as germination, morphology and responses to biotic and abiotic stress. Despite wide conservation in plants, a number of miRNAs are lineage specific. We describe the first genome wide survey of Eucalyptus miRNAs based on high throughput sequencing.

Results: In addition to discovering small RNA sequences, MIR loci were mapped onto the reference genome and interspecific variability investigated. Sequencing was carried out for the two most world widely planted species, E. grandis and E. globulus. To maximize discovery, E. grandis samples were from BRASUZ1, the same tree whose genome provided the reference sequence. Interspecific analysis reinforces the variability in small RNA repertoire even between closely related species. Characterization of Eucalyptus small RNA sequences showed 95 orthologous to conserved miRNAs and 193 novel miRNAs. In silico target prediction confirmed 163 novel miRNAs and degradome sequencing experimentally confirmed several hundred targets. Experimental evidence based on the exclusive expression of a set of small RNAs across 16 species within Myrtaceae further highlighted variable patterns of conservation and diversity of these regulatory elements.
\end{abstract}

Conclusions: The description of miRNAs in Eucalyptus contributes to scientific knowledge of this vast genre, which is the most widely planted hardwood crop in the tropical and subtropical world, adding another important element to the annotation of Eucalyptus grandis reference genome.

Keywords: Deep sequencing, Eucalyptus, Genome mapping, Interspecific variability, micro RNAs, Myrtaceae, Small RNAs

\section{Background}

In the last decade, small non-coding RNAs have emerged as key endogenous regulatory elements in eukaryotic cells. It became clear that part of the so called junk DNA was transcribed into silencing RNAs that take part in an intricate gene regulatory network with highly specific functions [1-3]. Among a variety of functional non coding small RNAs (sRNAs), two main classes have been subject

\footnotetext{
* Correspondence: marilia.pappas@embrapa.br

${ }^{1}$ Embrapa Recursos Genéticos e Biotecnologia, Brasília, DF, Brazil

Full list of author information is available at the end of the article
}

of intensive investigation in plants: small interfering RNAs (siRNAs) and micro RNAs (miRNAs). Mature siRNAs are predominantly 24-nt long transcribed by RNA polymerase IV with subsequent double strand synthesis $[4,5]$. They are fundamental players in cellular defense mechanisms against viruses [6,7] and epigenetic regulation, as the drivers of RNA directed DNA methylation (RdDM), contributing to the silencing of transposable elements and transcriptional gene silencing $[8,9]$. MiRNAs are 20 to 24-nt long sRNAs involved predominantly in posttranscriptional gene regulation. Single stranded miRNAs 
primary precursors - pri-miRNAs - are transcribed by RNA polymerase II from MIR genes [4], and processed by Dicer like 1 enzyme (DCL1) to generate an intermediary precursor (pre-miRNA), typically folded into a stable single strand stem loop structure [1, 10-12]. In plants, the mature miRNA is excised from the pre-miRNA, exported from the nucleus and then incorporated into protein RNA induced silencing complex (RISC). RISC is guided to the target messenger RNA (mRNA) by sequence complementarity to miRNA $[13,14]$. The silencing mechanism is determined by the degree of complementarity between the pair miRNA-target mRNA. Pairing mismatches are frequent in metazoans and miRNAs tend to block target mRNA by hindering its translation whereas high complementarity usually directs mRNA degradation in plants [15]. A wide variety of processes such as plant development [16-19], flowering [18, 20, 21], meristem and vascular differentiation [16], disease resistance [22-24] and response to abiotic stress [25-27] are regulated by miRNAs.

One of the first steps to ascertain how these components engage in complex regulatory networks is the characterization of the small RNA repertoire of a species. In recent years, second generation sequencing provided the technical breakthrough for rapid and comprehensive small RNA discovery including non-conserved and low abundance miRNAs [18, 26-36]. Accordingly, a surge in studies characterizing plant sRNAs was initiated. Despite the vast number of sRNA reads in sequencing data, bona fide plant miRNA genes of a given species are typically numbered in hundreds exemplars. Then extensive postprocessing becomes fundamental to adhere sRNA sequences to a strict set of rules defining miRNA genes, which have foundations in recalling their biogenesis mechanisms [37].

There are many examples of highly conserved miRNAs families in plants, some present from basal plant species to angiosperms [38]. However it is known that there is great interspecific variability in small RNAs repertoire with several MIR genes lineage or species specific. Studies on miRNA discovery, either by RNA sequencing or in silico prediction, have shown this high diversity in different species [39-42].

Among plant miRNAs present in the database miRBase [43], forest and fruit trees still have very few representatives aside from Poplar (Populus trichocarpa) [44]. Recent miRNA discovery studies were published for apple [45], Pinus densata [46], the rubber tree Hevea brasiliensis [47], peach [48], Chinese fir [35], Carya cathayensis [18] and Eugenia uniflora [34].

Eucalyptus is a highly diverse genus of the Myrtaceae family. Native to Australia and its northern islands, Eucalyptus species occur predominantly in the southern hemisphere from sea level to alpine tree line and from high rainfall to semi-arid zones [49]. Eucalyptus species are mostly outcrossers $[50,51]$ and their extensive genetic variation has been widely used in breeding programs [52]. Due to their noteworthy high growth rate, wide adaptability, high biomass production and carbon sequestration capabilities, eucalypts became the hardwood crop most widely planted in tropical and subtropical areas, exceeding 20 million hectares around the world [53]. Eucalyptus globulus and Eucalyptus grandis are currently the most extensively planted species among the $\sim 700$ species described for the genus, widely used as sustainable short fiber source for pulpwood and energy. Despite the great wood quality of E. globulus for pulp production, its temperate origin implies poor adaptation to highly productive tropical environments where $E$. grandis is the species of choice, making this latter one the most widely planted Eucalyptus species.

The genome of E. grandis was recently sequenced [54] and is available at Phytozome [55]. As part of that landmark development we performed a comprehensive annotation of miRNA genes, now fully described in this report. This additional layer of information will be valuable to promote a deeper genomic understanding of a number of processes such as tree growth, vascular development, phase change and response to environmental stresses, pests and pathogen, where miRNAs are known to be involved, and that currently make up the bulk of investigation both in forest productivity and health. Complementary strategies were used, starting with Illumina-based high throughput small RNA library sequencing (smRNASeq) for E. grandis and E. globulus, followed by degradome sequencing analyses for large-scale target mRNA identification. This study represents the first genome wide discovery, mapping and characterization of Eucalyptus miRNAs and should provide useful fundamental information for upcoming studies on gene regulation in what has now been promoted to an additional model species for forest tree genomics.

\section{Results}

\section{Small RNA sequencing data}

Illumina GAII deep sequencing was carried out for the small RNA fraction of four samples: one leaf (E. grandis BRASUZ1) and three developing xylem samples (E. grandis BRASUZ1 and two E. globulus trees). This experiment resulted in a total of $6,104,498$ raw reads ranging from $1,115,404$ to $1,766,355$ per sample. Pre-processing steps, namely quality screening, adapter and redundancy removal, resulted in a total of $1,857,986$ unique sequences (Table 1). Contaminant sequences of ribosomal, chloroplast and tRNA origin were filtered out totaling $1.8 \%$ of the unique sequences. 
Table 1 Overall sequencing counts of small RNA sequecing in Illumina GAll platform

\begin{tabular}{llll}
\hline Sample & $\begin{array}{l}\text { Total } \\
\text { raw reads }\end{array}$ & $\begin{array}{l}\text { Filtered } \\
\text { reads }\end{array}$ & $\begin{array}{l}\text { Unique } \\
\text { sequences }\end{array}$ \\
\hline E. grandis BR1 - leaf & 1.484 .867 & 1.450 .215 & 265.579 \\
E. grandis BR1 - xylem & 1.766 .355 & 1.695 .542 & 377.958 \\
E. globulus A2 & 1.737 .872 & 1.703 .806 & 377.958 \\
E. grandis C3 & 1.115 .404 & 1.093 .630 & 476.080 \\
Total & 6.104 .498 & 5.943 .193 & 1.874 .515 \\
\hline
\end{tabular}

Filtered reads refer to number of reads after filtering for low quality and no adapter sequences

Read size distribution shows the expected two main peaks at 21 and 24 nucleotides (nt). Twenty-four nucleotide sequences are the most abundant reads in all four samples (Fig. 1). As seen by size ranking of smRNA-Seq data, 24-nt sequences also show extensive sequence diversity with the highest number of unique sequences (clusters) (Additional file 1: Figure S1). Despite the high diversity, each 24-nt cluster exhibits low expression level - none of the 24-nt read makes up $1 \%$ of the total counts in the size class. Twenty-one nucleotide sequences show an opposite distribution showing less sequence diversity (fewer unique sequences) but the highest counts per cluster observed amongst all sRNA size classes analyzed (from 15 to 28nt). The most abundant sequence within the 21-nt class varies from $10 \%$ up to $40 \%$ of total counts in BRASUZ1 leaves sample (Fig. 2).

Conserved miRNAs were often observed among 21-nt reads with highest counts. MiR159a was consistently the most abundant 21-nt sequence in three out of four samples $-E$. grandis BRASUZ1 leaves (40 \%) (Fig. 2a) and developing xylem (10.5 \%) (Fig. 2b), and E. globulus C3 developing xylem (18.2 \%) (Fig. 2d). In the E. globulus A2 xylem sample, miR159a (6.99\%) was outnumbered by miR172g $(9.16 \%)$, but was the second most abundant 21-nt read (Fig. 2c). MiR159a is a highly conserved miRNA as seen by alignment of Eucalyptus miR159a with all plant orthologs present in miRBase (Fig. 3). MiR166 and miR396 are other conserved miRNAs that are featured among the top fifteen 21-nt reads in all four samples. A broad search for conserved miRNAs was carried out and is discussed further.

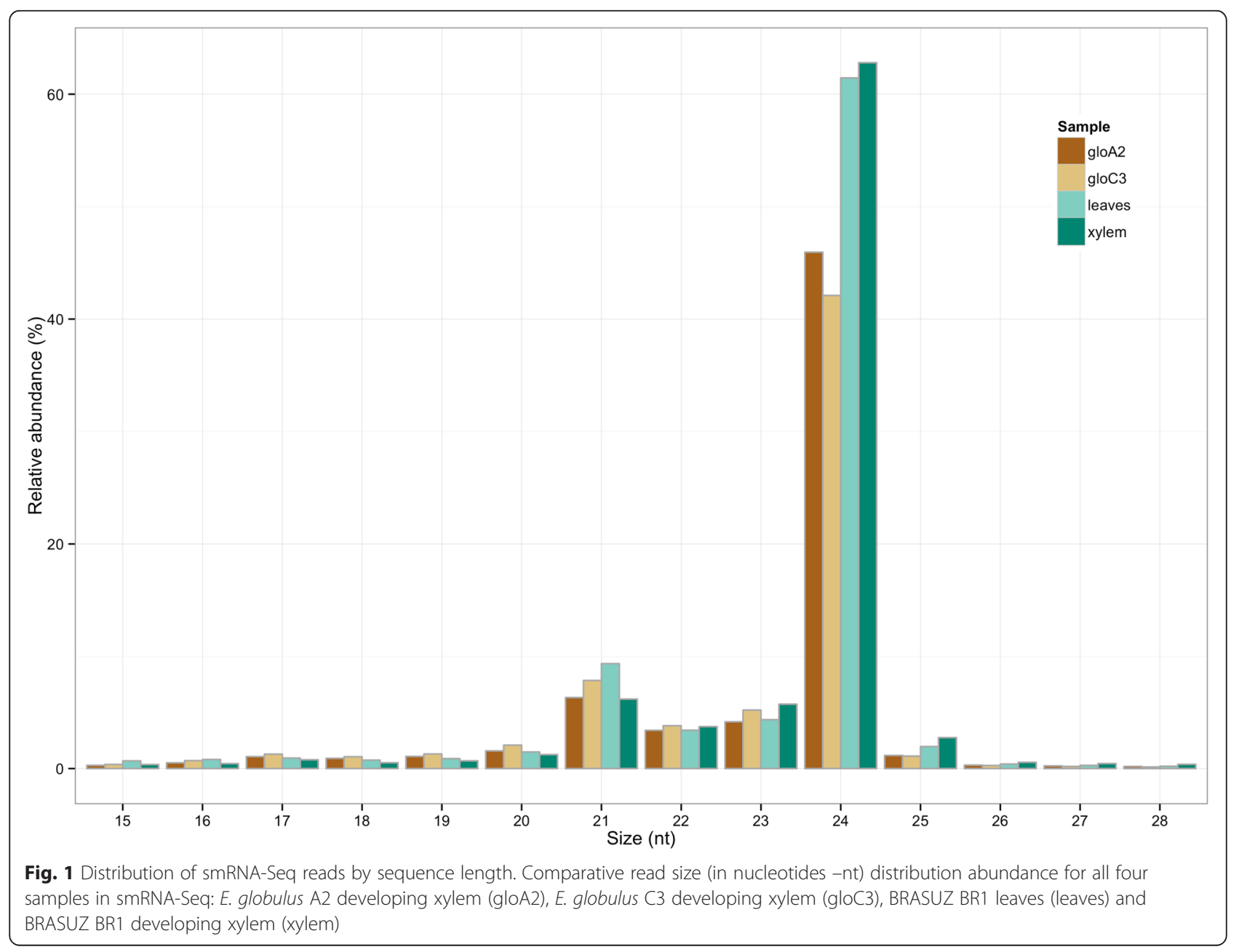




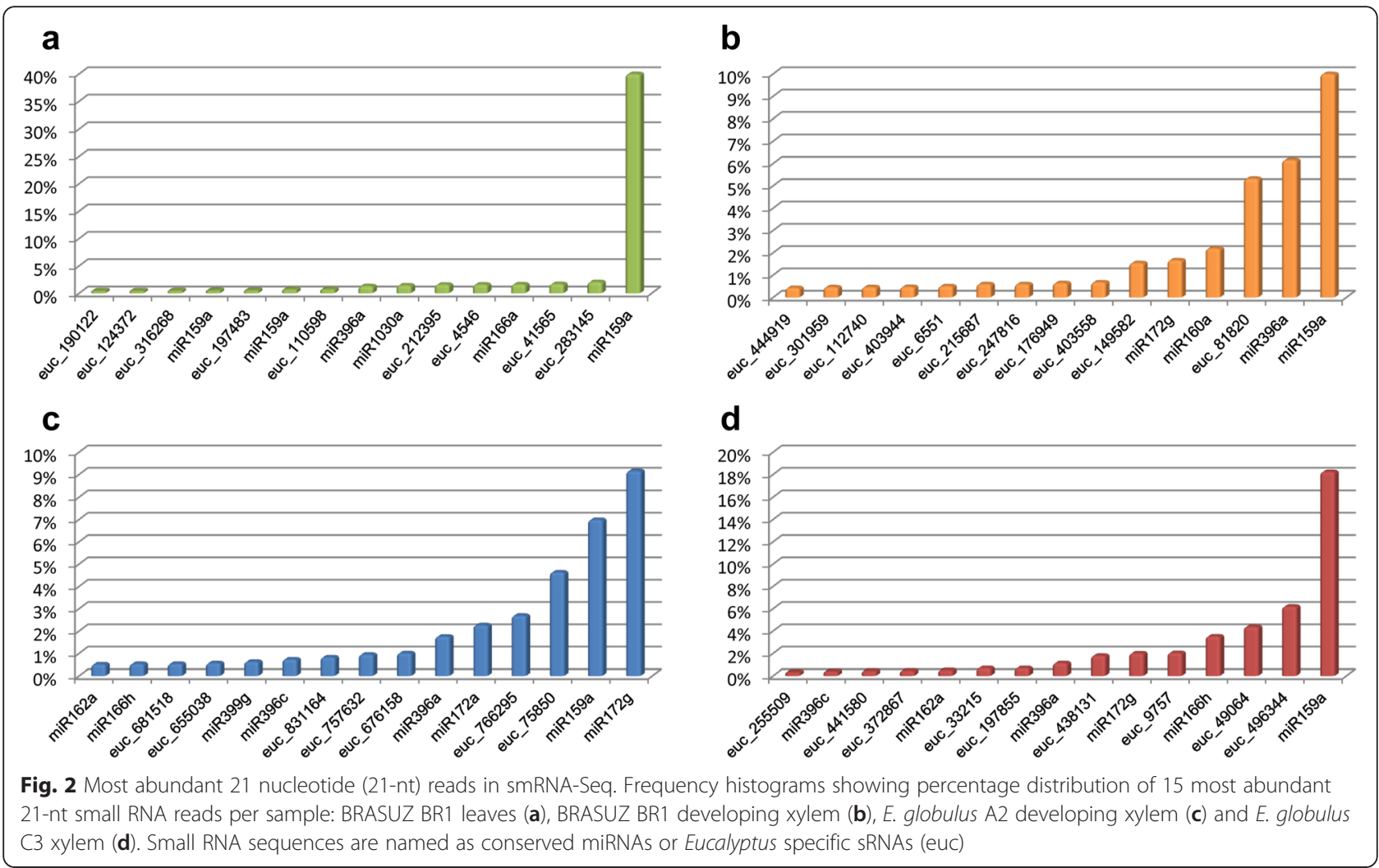

egr-miR159a UUUGGAUUUAAGGGAGCUCUA
osa-miR159a UUUGGAUUGAAGGGAGCUCUG
tae-miR159a UUUGGAUUGAAGGGAGCUCUG
zma-miR159a UUUGGAUUGAAGGGAGCUCUG
sof-miR159a UUUGGAUUGAAGGGAGCUCUG
ssp-miR159a UUUGGAUUGAAGGGAGCUCUG
hvu-miR159a UUUGGAUUGAAGGGAGCUCU-
ptc-miR159a UUUGGAUUGAAGGGAGCUCUA
vun-miR159a UUUGGAUUGAAGGGAGCUCUA
aly-miR159a UUUGGAUUGAAGGGAGCUCUA
ath-miR159a UUUGGAUUGAAGGGAGCUCUA
gma-miR159a UUUGGAUUGAAGGGAGCUCUA
mtr-miR159a UUUGGAUUGAAGGGAGCUCUA
bra-miR159a UUUGGAUUGAAGGGAGCUCUA
pta-miR159a -UUGGAUUGAAGGGAGCUCCA
vvi-miR159a CUUGGAGUGAAGGGAGCUCUC

Fig. 3 Conservation of miR159a. Multiple sequence alignment of Eucalyptus grandis miR159a (egr-miR159a) and plant miR159a sequences from miRBase (sequence id as in miRBase)

\section{Genome mapping}

Mapping of $1,857,986$ sRNA unique sequences on the $E$. grandis reference genome (Phytozome 8.0) was carried out using BWA [56]. Uniquely mapped reads per sample varied from $67.2 \%$ to $87.3 \%$ (Table 2). Mapping data confirmed the high diversity of the 24-nt sRNA sequences previously mentioned in total reads counting. For all samples, 24-nt reads represent the highest number of uniquely mapped sequences.

Mapping data was investigated for correlation between sequence size and mapping location in repetitive regions. Mapping data of unique reads from 19 to 26-nt showed sRNA reads located mostly in repeat regions $(70.1 \%$ in E. grandis BRASUZ1 leaves, $59.3 \%$ in E. grandis BRASUZ1 xylem, $64.3 \%$ in E. globulus A2 xylem and $64.9 \%$ in E. globulus C3 xylem). Size distribution of mapped reads revealed a general tendency to mapping on repetitive regions irrespective of the sequence size (Fig. 4).

\section{Characterization of conserved miRNA sequences}

A similarity search of all 20 to 22 nucleotides unique sequences was done using PatMan [57] against miRBase plant mature miRNA sequences. A total of 303 distinct 21-nt sRNAs showed significant similarity (with at most three mismatches) to an orthologous miRNA sequence in miRBase. Conserved reads with $100 \%$ identity totaled 95 sequences encompassing 25 
Table 2 Mapping data of unique sequences from smRNA-Seq to the Eucalyptus grandis genome using BWA

\begin{tabular}{lllll}
\hline Sample & BR1 leaves & BR1 xylem & E. globulus A2 & E. globulus gloC3 \\
\hline Mapped sequences & $231.893(87,3 \%)$ & $327.580(86,7 \%)$ & $507.430(67,2 \%)$ & $321.403(67,5 \%)$ \\
\hline
\end{tabular}

miRNA families (Additional file 2: Table S1). Expression of miRNAs miR156a, miR159a, miR160 and miR172b was assessed by northern blot in leaves and developing xylem of E. dunnii, E. urophylla and $E$. grandis BRASUZ1 (Additional file 3: Figure S2).

MiRNAs with 22-nt in length constitute a class of sRNAs not as abundant as the 21-nt ones but which make up a subclass with outstanding role in triggering biosynthesis of phased secondary siRNAs known as tasiRNAs (trans-acting siRNAs) or phasiRNAs (phased siRNAs) [58, 59]. Sixteen 22-nt conserved miRNA sequences with $100 \%$ identity and 69 with up to three mismatches were identified.

\section{In silico prediction by miRDeep}

There are hundreds of MIR genes identified in plant genomes. The massive bulk of smRNA-Seq reads thus requires careful analysis to identify bona fide miRNA genes, as established by a set of strict criteria [37]. The first one is to be excised from a stem loop arm of a single stranded intermediary precursor (pre-miRNA). To test smRNA-Seq data for this criterion, the reference genome of E. grandis BRASUZ1 provided the ideal conditions for a broad computational search. We used miRDeep2 package for de novo prediction of miRNAs from sequencing data [60]. After a genome-wide search for candidate regions complying with precursor secondary structure constraints, 193 mapped sequences showed to be compatible with a MIR gene locus (Additional file 4: Table S2). Eighty two of these in E. grandis BRASUZ1 leaves sample, 55 in E. grandis BRASUZ1 xylem, 74 and 73 in E. globulus A2 e C3 xylem samples, respectively.

Five of the most abundant 21-nt reads in smRNA-Seq data (euc_sRNA_149582, euc_sRNA_75850, euc_sRNA_ 438131, euc_sRNA_33215 and euc_sRNA_372867) (Fig. 2) had their expression experimentally revalidated by northern blot in three Eucalyptus species - E. dunni, E. urophylla and E. grandis (Additional file 5: Figure S3). Nevertheless,

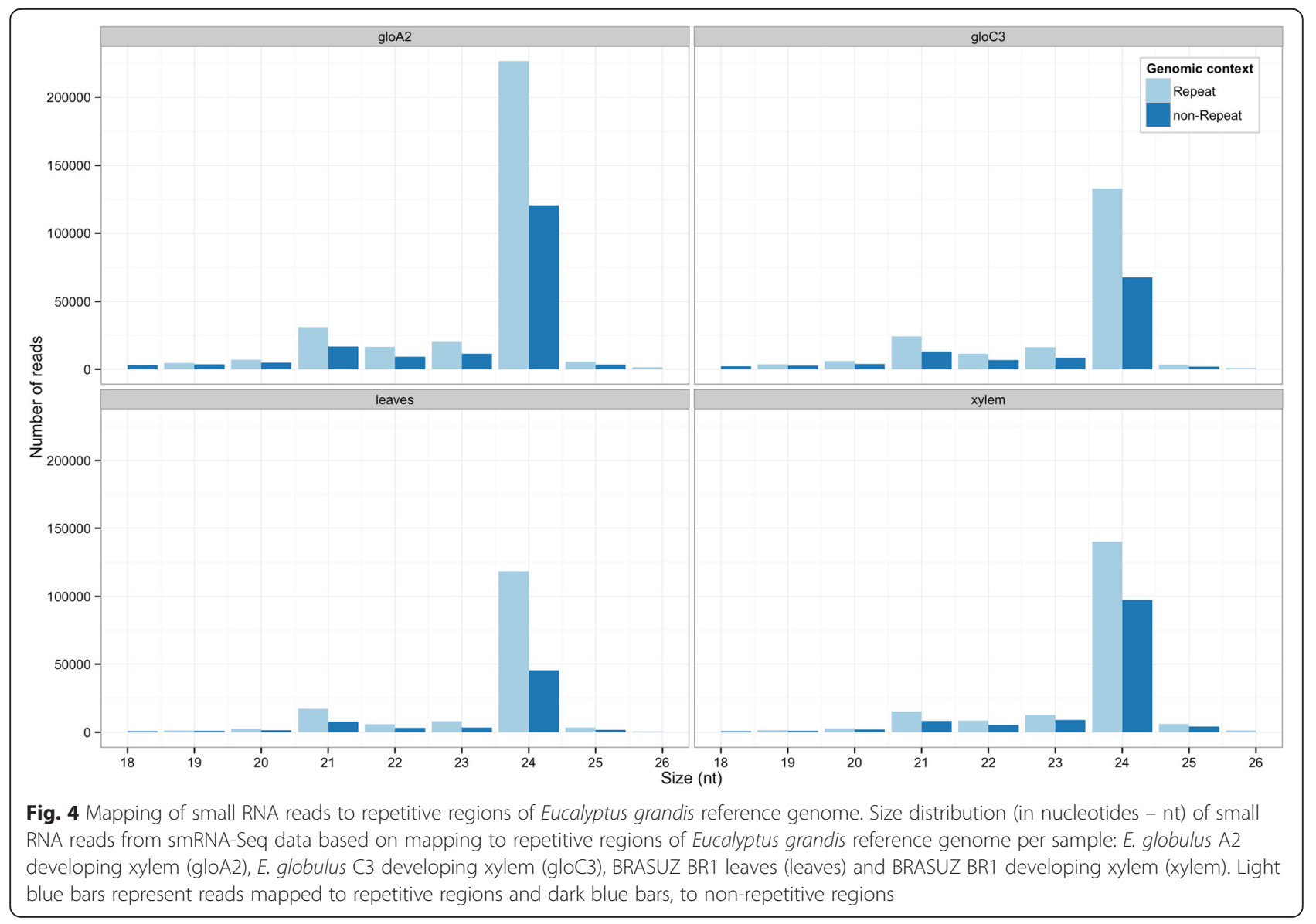


despite the evidences of expression (smRNA-Seq and blot) and mapping on the reference genome of $E$. grandis, their flanking regions did not meet the requirements for a typical miRNA precursor structure.

\section{Target prediction and degradome sequencing}

Prediction of transcripts targeted by miRNAs adds another level of in silico confirmation, providing clues about potential biological processes being regulated. Target prediction was performed for all mapped sequence candidates with a compatible precursor secondary structure using psRNATarget [61]. The Eucalyptus transcript database from Phytozome was used for reverse complementary matching between smRNA-Seq data and potential target transcripts. Functional annotation of targets was retrieved from the BioMart tool available in Phytozome. The number of predicted targets for each miRNA candidate varied from one to twenty transcripts. The enrichment for specific protein domains in predicted target mRNAs is shown in Fig. 5 and indicates the prevalence of signature domains for NB-LRR (NB-ARC, leucine rich repeat) disease resistance genes, ion transporters, SBP (squamosa binding proteins) transcription factors and PPR (pentatricopeptide repeat) proteins. Predicted targets also include transcripts related to wood formation such as cellulose synthases and cytochrome P450 which is involved both in biosynthetic and detoxification pathways.

Target prediction for miR159a, the most abundant 21-nt read in our smRNA-Seq data, resulted in 10 predicted transcripts (Additional file 6: Table S3) including two MYB transcription factors (Eucgr.G03183.1 and Eucgr.E01581.1).
To get a glimpse of miRNA binding site conservation on MYB transcription factors, the orthologs were searched in Phytozome database, the transcripts scanned for the presence of miR159a binding site, using psRNATarget, and the ones with at least 20 bases flanking the binding the site were used to create a sequence logo (using WebLogo 3 [62]) highlighting the miRNA binding site at the target transcripts (Fig. 6).

To experimentally confirm in silico predicted targets, a degradome sequencing experiment was performed. Libraries of 3' cleavage products were prepared from mRNA samples of leaf and developing xylem from an adult E. grandis BRASUZ1 tree. Illumina HiSeq sequencing of both samples resulted in 27,244,395 raw reads. After filtering for low quality reads, including no adapter, no insert and sequences smaller than 18 nucleotides, the total number was reduced to $26,387,851$ (97.53 \%) valid sequence reads. Annotation of filtered reads resulted in $18,257,616$ total and 2,887,536 unique sequences. Non coding RNAs - such as ribosomal, transporter, small nuclear and small nucleolar - accounted for around $2.67 \%$ of the total read count. Sharing of unique sequences between the samples represented $9.64 \%$ of the reads.

Analysis of degradome sequencing data with CleaveLand pipeline identified 189 cleaved transcripts targeted by 21-nt sRNA sequences from leaves and 324 from xylem smRNA-Seq data (Additional file 7: Table S4; Additional file 8: Table S5). Considering the 22-nt sRNAs, the number of pairs was 149 and 248, respectively. Targets included a set of transcription factors such as MYB, GRAS and SBP families, cation transporter genes and ARFs (auxin response factors) as reported

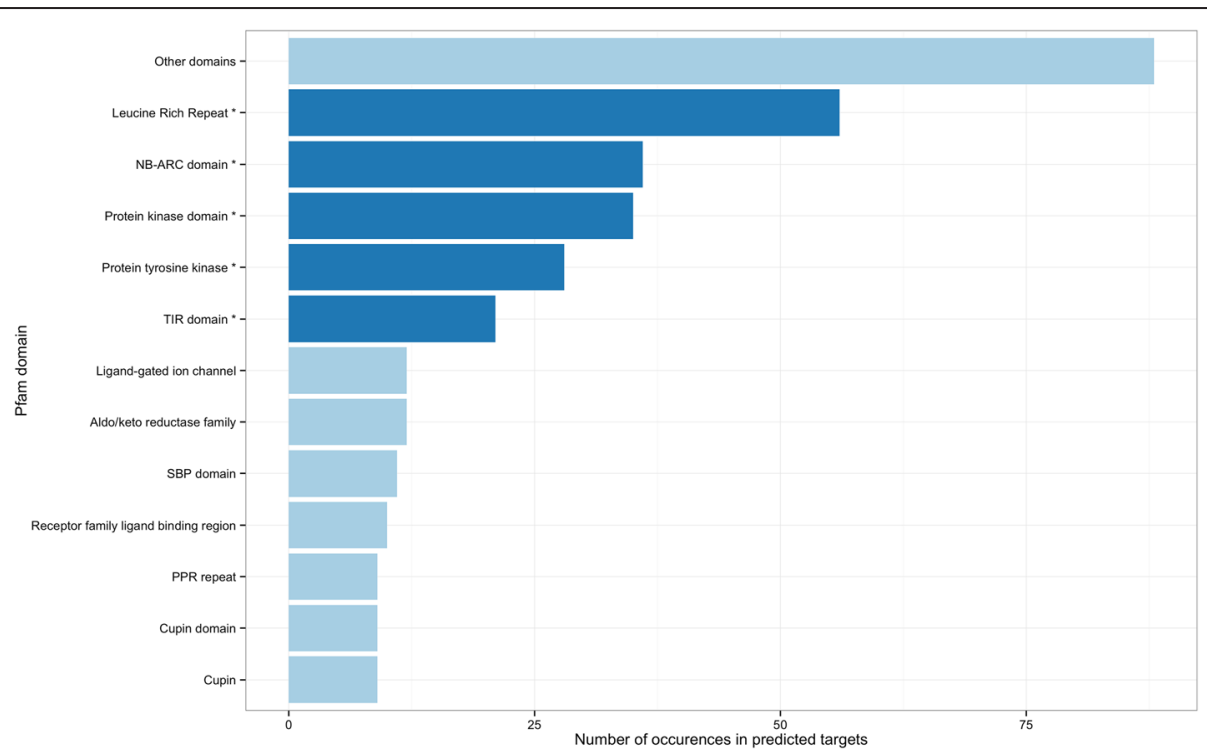

Fig. 5 Enrichment of protein domains in predicted micro RNA targets. Count of protein domains in messenger RNA targets predicted by psRNATarget. Protein domains typical from disease resistance genes family TIR-NB-LRR $(*)$ are the most abundant 


\section{miR159a}

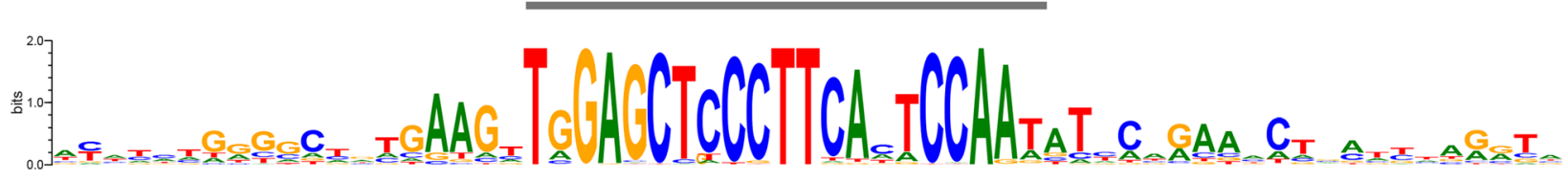

Fig. 6 Sequence logo of MYB transcription factor transcripts retrieved from Phytozome 10.3 highlighting miR159a binding site (created with WebLogo 3). Each transcript is shown with 20 nucleotides flanking the binding site

before for other plant species $[63,64]$ and matching the in silico results predicted by psRNATarget.

A wide variety of other transcripts involved in diverse physiological processes such as cytochrome P450, TIRNB-ARC disease resistance genes and nuclear transport factor 2 (NTF2) were also identified in the degradome data. Some results corroborate known targets of conserved miRNAs such as a SBP transcription factor cleaved by miR156 [65-67], an ARF transcript, by miR160 [68, 69] and the transcription factor AP2 (apetala 2), by miR172 $[70,71]$. The degradome sequencing data further supported two newly identified Eucalyptus miRNAs, which displayed two distinct transcripts of cellulose synthase as targets, detected only in the xylem sample (Eucgr.D00476.1 and Eucgr.H00939.1 in Phytozome).

\section{Conservation within Myrtaceae}

Interspecific variability in sRNA loci was observed from mapping data of E. grandis and E. globulus sRNA reads on the $E$. grandis reference genome. In our smRNA-Seq data, the number of mapped sequences differed by $20 \%$ between the two species (Table 2). In order to extend the inquiry of sRNA conservation within Myrtaceae, we expanded the analysis to other species and genera. A comparative analysis of Eucalyptus and Eugenia uniflora smRNA-Seq data (available at the NCBI Gene Expression Omnibus, GEO, accession number GSE38212) was carried out. Mapping of sRNA reads of E. uniflora to the E. grandis genome totaled 1,392,334 (34.7\%) of the total unique sequences. Size sorting of mapped sequences showed a higher conservation of 21-nt sRNAs and lower of $24-\mathrm{nt}, 61.6 \%$ and $21.1 \%$ respectively (Table 3). Same tendency was observed when overall unique sequences were compared. Considering only perfect matches
(100\% identity), 21-nt common sequences were 10 fold higher than 24-nt and allowing 1 mismatch, 7 fold higher. Higher conservation was also observed for 22 nucleotides sRNAs when compared to 24 nucleotides.

Northern blot analysis of some of the most expressed sRNA reads in the smRNA-Seq data was carried out in order to investigate conservation within the Myrtaceae family. Five sRNAs probes were hybridized against RNA from completely developed leaves of seventeen Myrtaceae species, including six Eucalyptus species (E. grandis, E. botryoides, E. brassiana, E. globulus, E. pellita and $E$. resinifera), a hybrid $E$. urograndis ( $E$. urophylla $\mathrm{x} E$. grandis), in addition to ten other species of different genera of Myrtaceae - Corymbia citriodora (previously classified as Eucalyptus citriodora), Eugenia uniflora, Psidium cattleyanum., Psidium guajava, Syzygium cumini, Melaleuca lateritia, Eugenia calycina, Eugenia dysenterica, Campomanesia pubescens and Syzygium malaccense. Three diverse selected outgroup species - Glycine max, Lycopersicum esculentum and the gymnosperm Pinus taeda - were also included in the experiment. Expression of two sRNAs (euc_sRNA_149582, euc_sRNA_ 75850) were not detected in any of the outgroup species (Fig. 7a) but were consistently detected in all Myrtaceae samples (Fig. 7b). The other three sRNAs (euc_sRNA 438131, euc_sRNA_33215 and euc_sRNA_372867) are potentially Eucalyptus specific as their expression were confirmed in three Eucalyptus species - E. grandis, E. dunnii and E. urophylla - (Additional file 5: Figure S3) but not in the outgroups G. max, O. sativa, L. esculentum and P. taeda nor in the other Myrtaceae species tested - C. citriodora and E. uniflora (Fig. 7a). Secondary structure prediction for genome flanking regions of these sRNAs failed to confirm them as miRNAs.

Table 3 Comparative analysis of Eucalytpus and Eugenia uniflora small RNA sequencing data

\begin{tabular}{llll}
\hline Sequence lenght $(\mathrm{nt})$ & Eugenia and Eucalyptus overall common sequences & Eugenia sequences mapped to E. grandis & Eugenia unique sequences \\
\hline 21 & $7,018(1.2 \%)$ & $352,691(61.6 \%)$ & 572,311 \\
22 & $4,742(1.7 \%)$ & $121,025(42.9 \%)$ & 282,335 \\
24 & $5,231(0.2 \%)$ & $514,683(21.1 \%)$ & $2,436,699$ \\
Total & $16,991(0.5 \%)$ & $988,399(30.0 \%)$ & $3,291,345$ \\
\hline
\end{tabular}

Number of unique sequences common to both E. uniflora and Eucalyptus (no mismatches), number of mapped unique sequences of $E$. uniflora small RNAs on the $E$. grandis genome and total number of $E$. uniflora unique sequences 


\section{a}

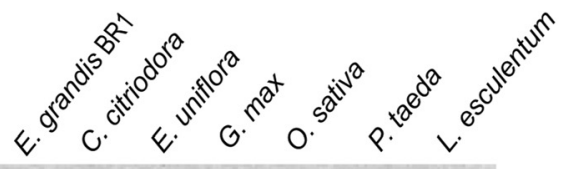

euc_sRNA_149582

euc_sRNA_75850

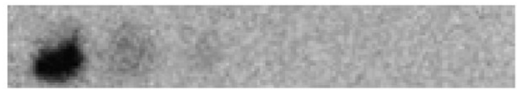

euc_sRNA_438131

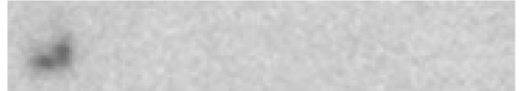

euc_sRNA_33215

euc_sRNA_372867

$\operatorname{miR} 319$
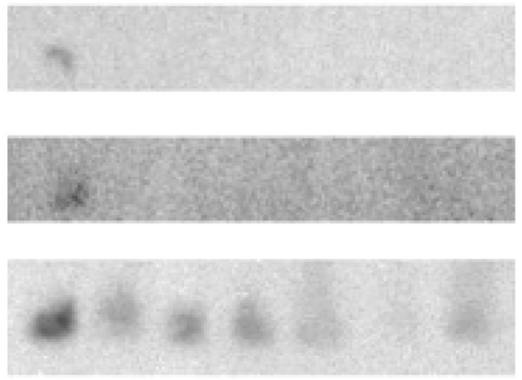

snRNA U6

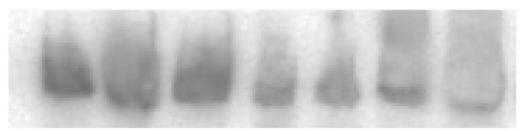

b

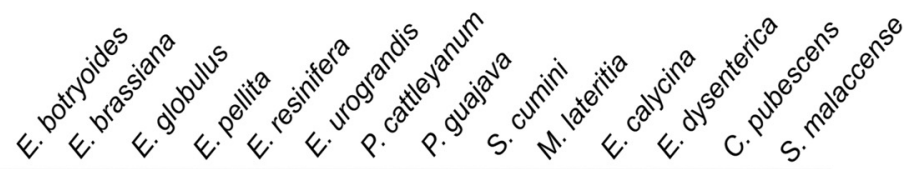

euc_sRNA_149582

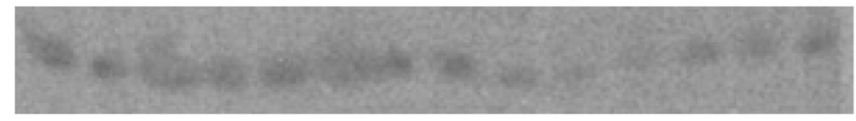

euc_sRNA_75850

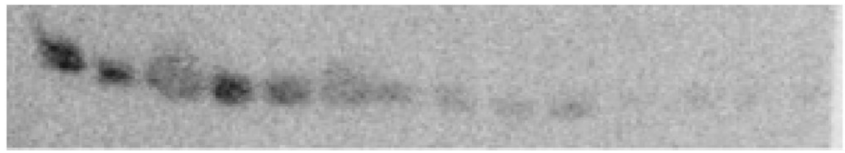

miR319

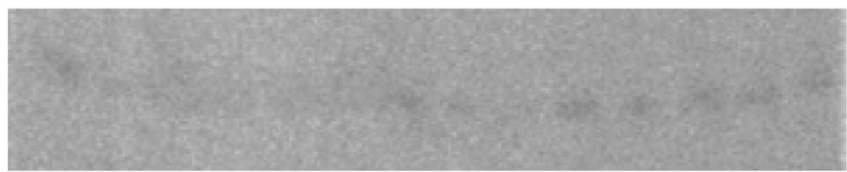

snRNA U6

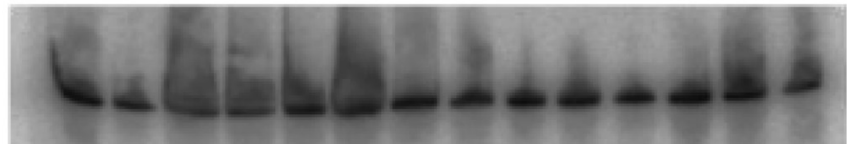

Fig. 7 Conservation of small RNAs within Myrtaceae family species and unrelated outgroups via expression validation. Northern blot analysis of some of the most abundant 21-nt sRNAs in smRNA-Seq was assessed in leaves of Eucalyptus grandis BRASUZ1 (E. grandis BR1), Corymbia citriodora, Eugenia uniflora, Glycine max, Oryza sativa, Pinus taeda and Lycopersicum esculentum (a); Eucalyptus botryoides, E. brassiana, E. globulus, E. pellita, E. resinifera, E. urograndis (hybrid of E. urophylla x E. grandis), Psidium cattleyanum, Psidium guajava, Syzygium cumini, Melaleuca lateritia, Eugenia calycina, Eugenia

dysenterica, Campomanesia pubescens and Syzygium malaccense (b). Micro RNA miR319 was blotted as positive control and snRNA U6 as loading control 


\section{Discussion}

Our results highlight the pivotal importance of a careful analysis and raw data filtration of the massive amounts of sequence data produced by next generation sequencing. The large initial number of reads is progressively reduced after consecutive analyses. Ultimately, 1,405,134 ( $75.63 \%$ of total valid reads) 21 -nt unique sequences mapped unambiguously to the E. grandis BRASUZ1 reference genome. Ninety five 21-nt unique sequences are conserved micro RNAs with $100 \%$ identity to sequences available in miRBase. Three hundred and three have an orthologous sequence in miRBase with up to 3 mismatches and thus potentially correspond to new isoforms of conserved miRNA families. While the identification of conserved miRNAs can be easily accomplished by similarity search with previously described orthologous sequences, the annotation of new miRNAs requires the adherence to a set of strict criteria such as the presence of a compatible secondary structure of precursor sequence and target prediction. This process was highly benefited by the availability of a high quality $E$. grandis reference genome, possibly the last one generated exclusively by Sanger sequencing. Among all 21-nt mapped sequences, 193 have a compatible miRNA precursor secondary structure as predicted by miRDeep2. From this subset, 16321 nt sequences had at least one predicted target by psRNATarget, complying with the criteria to annotate a miRNA.

As seen by the read size distribution, 24-nt reads were the most abundant size class in the smRNA-Seq data with up to 3.75 times the number of 21-nt reads. The predominance of 24-nt sRNAs, mainly represented by siRNAs, is well known in angiosperms and had been previously reported for several species [4, 72-74]. Noteworthy is that, in addition to being the most abundant class overall, 24-nt sRNA Eucalyptus sequences constitutes by far the most diverse group, with the greatest number of clusters. The 24-nt overall high sequencing read counts observed is therefore explained by its diversity of unique sequences even with few reads per cluster when compared to 21-nt reads. Altogether this is in agreement with the premise that small RNA repertoire in plants is dominated by a vast number of 24-nt small interfering RNAs (siRNAs) [15]. Contrary to that, 21-nt reads - which include both siRNAs and miRNAs - are less diverse, with fewer unique sequences, but exhibit the highest read counts per cluster in sequencing data.

As heterochromatic 24-nt sRNAs predominantly silence transposons and repeat regions by directing DNA methylation at complementary sites in the genome [15], one would expect to see a higher proportion of these sRNAs mapping to repetitive regions. In fact this seemed to be an overall tendency for all Eucalyptus sRNAs from 19 to 26-nt as observed for all samples in the smRNA-Seq.
Interspecific variability in small RNA content of the genome was evidenced by the mapping data. E. grandis BRASUZ1 displayed $20 \%$ higher proportion of uniquely mapped sequences to its own genome when compared to E. globulus. Pairwise comparison of smRNA-Seq data for all samples corroborated this assertion: the most similar samples were from developing xylem of different trees of E. globulus. Pairwise comparison also highlighted the tissue specificity of sRNA expression, as the most diverse repertoire of sRNA reads were from distinct tissues- developing xylem and leaves - collected simultaneously from the same E. grandis BRASUZ1 tree (data not shown).

It is suggested that conserved miRNAs usually have higher expression and that lineage specific ones are expressed at lower levels or in specific tissues, developmental stages or conditions [29, 75]. Counting of our 21-nt sequences reinforced this concept, as conserved miRNAs were consistently seen among the most abundant reads. Though a large number of 21-nt sRNAs that are not conserved miRNAs were also highly expressed, in silico analysis of these sequences showed that they do not fit canonical miRNA criteria being representatives of another class of sRNAs.

Recently, a large scale identification of miRNAs was performed in Eugenia uniflora [34]. A comparative analysis between smRNA-Seq data from Eucalyptus and E. uniflora indicated a high conservation within Myrtaceae family. This high conservation probably arises mostly from conserved miRNAs sequences as evidenced by higher conservation of 21-nt sequences. MiRNAs commonly have non-related targets and are frequently involved in housekeeping conserved pathways. As miRNA silencing relies on sequence complementarity to heterologous targets, this class tends to suffer more selective evolutionary pressure. On the other hand, siRNAs usually silence related targets or even their own origin loci, acting in cis. It is suggested that siRNAs suffer little or no selective pressure to maintain sequence conservation resulting in high evolutionary rates [76]. Our experimental results of northern blot for 21-nt sRNAs (other than miRNAs) assessed in various Myrtaceae species and outgroups suggested a tendency of sequence conservation of highly expressed sRNAs within the family.

Target prediction showed predominance of NB-LRR proteins, the most common disease resistance genes in plants, known to be highly regulated by sRNAs [77] Transcription factor families, as SBP and MYB, were also abundantly present in target prediction. MYB proteins are known as transcription factors related to wood formation [78]. The R2R3-MYB gene family, as an example, is supposed to control lignification during xylogenesis (wood growth) [79, 80]. Targets involved in biosynthetic pathways were also predicted such as cytochrome $\mathrm{P} 450$. These proteins play a key role in the 
synthesis of structural polymers as lignins [81] which together with cellulose are the two basic wood components.

A recent study on Eucalyptus grandis miRNAs investigated the relationship between alterations in miR156 and miR172 expression, associated with vegetative phase change $[19,82,83]$, and adventitious root induction during development [84]. Inquiries like this highlight the potential of miRNA investigation in diverse biological pathways with a vast impact on plant development and productivity among other aspects. That study also conducted a profile on miRNA expression in cuttings describing 40 known and 8 novel miRNAs, including one (Cluster_41475) also present in our data (Scaffold11 - 40625339-40625414).

\section{Conclusions}

This work provides the first comprehensive genome-wide discovery and characterization effort of miRNA in species of Eucalyptus. High throughput smRNA-Seq with in silico and experimental evidences allowed the characterization of conserved and novel miRNAs. Due to the lack of biological replicates in our smRNA-Sequencing data, an addition that would have allowed further quantitative analyses, we limited our study to a qualitative survey of miRNAs. Nevertheless, the data presented lays the foundation for forthcoming differential miRNA expression analyses.

The availability of a high quality genome sequence for E. grandis was a key asset to carry out a robust precursor structure prediction and this in turn provided improved experimental evidence to support the discovery of bona fide novel miRNAs. When a reference genome is not available, precursor secondary structure prediction is dependent on the availability of expressed sequence tags (ESTs) a method that has limitations. Furthermore, by using smRNA-Seq obtained from the same exact tree whose genome is the reference genome sequence, the analysis considerably improved the number of mapped sequences. The smRNA-Seq data from E. globulus on the other hand, provided solid evidence confirming the interspecific variability in the small RNA repertoire even between related species belonging to the same subgenus Symphyomyrtus. Availability of a large transcript database of the target species also highly favored the identification and characterization of targets. The identification, mapping and characterization of miRNAs loci described in this work directly contributed to the annotation of the Eucalyptus grandis genome [54] adding another layer of information to the current reference genome.

\section{Methods}

Plant material, RNA extraction and sequencing

Plant material for smRNA-Seq includes the same tree genotype used by JGI-DOE (Joint Genome Institute -
USA Department of Energy) for whole genome sequencing: E. grandis (BRASUZ1 tree), a selfed tree $\left(\mathrm{S}_{1}\right)$ from Suzano Group (Brazil). RNA from four biological samples was prepared for deep sequencing experiments: developing xylem and leaves of $E$. grandis BRASUZ1 and developing xylem of two unrelated $E$. globulus trees (named A2 and C3). RNA extraction was performed with an adapted CTAB protocol [85]. Library preparation for Illumina GAII sequencing used single end kit and barcoded adapters to multiplex samples in one lane run, all performed by Fasteris SA (Switzerland). For experimental validation via northern blot, developing xylem and leaves from two trees of E. dunnii and two of E. urophylla and leaves from two clones of $E$. grandis BRASUZ1 were employed. All RNA samples were obtained from fully developed leaves from adult trees.

\section{Analytical pipeline}

A custom-made computational pipeline was developed to process sRNA sequencing data. A pre-processing step cleans the sequences by trimming sequencing adaptors, quality screening and contaminant removal (including ribosomal, chloroplast and tRNA). Processed reads were size sorted, quantified (tag counting) and used to create a non-redundant read set using UCLUST [86]. The non-redundant reads were mapped to the $E$. grandis reference genome available in Phytozome [55] using BWA [56], with parameters $-\mathrm{n} 1$. In order to identify conserved miRNA sequences, a similarity search using PatMan [57] was carried out against the subset of plant-specific mature sequences from miRBase release 19 [43, 87], allowing at most three mismatches (paramenters -e 3). Novel miRNAs were predicted using the miRDeep2 pipeline [60] (mapper parameters $-\mathrm{e}-\mathrm{j}-\mathrm{l} 19-\mathrm{o}$ 16), which performs a genome wide search for potential miRNA precursors based on the extension of genome regions around mapped reads followed by secondary structure prediction and stability evaluation. Messenger RNA target prediction was performed using psRNATarget [61] applying default settings against the $E$. grandis transcript database from Phytozome server (version 8.0) [55].

\section{Degradome sequencing}

Total RNA from E. grandis BRASUZ1 developing xylem and leaves was used for parallel analysis of RNA ends (PARE) library preparation followed by Illumina HiSeq sequencing (BGI - Hong Kong). Analysis of degradome sequencing was performed by CleaveLand 3 pipeline that outputs potentially cleaved sRNA targets from both degradome and smRNA-Seq data [88]. 


\section{Additional files}

Additional file 1: Figure S1. Size rank profile of smRNA-Seq data. Smal RNA sequence clusters illustrating the number of clusters per sequence size (nt) and the abundance of reads in each cluster (dots). BRASUZ leaves (leaves), BRASUZ 1 developing xylem (xylem), E. globulus A2 developing xylem (gloA2) and E. globulus C3 xylem (gloC3). (TIFF 756 kb)

Additional file 2: Table S1. Annotation of Eucalyptus conserved miRNAs by similarity search against miRBase. (XLSX $33 \mathrm{~kb}$ )

Additional file 3: Figure S2. Northern blot analysis of conserved micro RNAs. (TIF $4319 \mathrm{~kb}$ )

Additional file 4: Table S2. Eucalyptus miRNA loci predicted by miRDeep2. (XLS 26 kb)

Additional file 5: Figure S3. Assessing small RNA conservation in Eucalyptus. (TIF $4142 \mathrm{~kb}$ )

Additional file 6: Table S3. Eucalyptus miR159a targets predicted by psRNATarget. (DOCX $12 \mathrm{~kb}$ )

Additional file 7: Table S4. Targets from degradome sequencing for BRASUZ1 21 bp RNAs - leaves. (XLS 30 kb)

Additional file 8: Targets from degradome sequencing for BRASUZ1 21 bp RNAs - xylem. (XLS $50 \mathrm{~kb}$ )

\section{Abbreviations}

AP2: Apetala 2; ARFs: Auxin response factors; ESTs: Expressed sequence tags; GEO: Gene Expression Omnibus; miRNA: micro RNA; mRNA: Messenger RNA; NB-LRR: NB-ARC, leucine rich repeat; nt: Nucleotides; NTF2: Nuclear transport factor 2; phasiRNA: Phased siRNA; PPR: Pentatricopeptide repeat; premiRNA: Intermediary miRNA precursor; pri-miRNA: Primary miRNA precursor; RdDM: RNA directed DNA methylation; RISC: RNA induced silencing complex SBP: Squamosa binding proteins; siRNA: Small interferent RNA; smRNASeq: Small RNA library sequencing; sRNA: Small RNA; tasiRNA: Trans-acting SiRNA.

Competing interests

The authors declare that they have no competing interests.

\section{Authors' contributions}

MCRP contributed with the design of the study, collected samples, carried out all the experimental studies and prepared the original draft of the manuscript. GPJr idealized the design of the study, performed bioinformatic analysis and contributed to the writing and revising of the manuscript. DG contributed with the design of the study and revised the manuscript. All authors read and approved the final manuscript.

\section{Acknowledgements}

We are grateful to Dr Scott Poethig for sharing the northern blot protocol, Simone Ribeiro for assistance with the northern blots, Alessandra Reis for technical assistance with the smRNA-Seq experiment and to Fasteris SA, especially to Laurent Farinelli, for the invaluable support. DG and GPJr are grateful for the financial support through CNPq research fellowships. This work was funded by Embrapa and by FAP-DF "NexTree" project grant.

\section{Author details}

${ }^{1}$ Embrapa Recursos Genéticos e Biotecnologia, Brasília, DF, Brazil.

2Universidade de Brasília, Brasília, DF, Brazil. ${ }^{3}$ Programa de Pós-Graduação em Ciências Genômicas e Biotecnologia, Universidade Católica de Brasília, Brasília, DF, Brazil.

Received: 19 June 2015 Accepted: 15 December 2015

Published online: 29 December 2015

\section{References}

1. Lagos-Quintana M, Rauhut R, Yalcin A, Meyer J, Lendeckel W, Tuschl T. Identification of tissue-specific microRNAs from mouse. Curr Biol. 2002;12(9):735-9.

2. Llave C, Kasschau KD, Rector MA, Carrington JC. Endogenous and silencingassociated small RNAs in plants. Plant Cell. 2002;14(7):1605-19.
3. Reinhart BJ, Weinstein EG, Rhoades MW, Bartel B, Bartel DP. MicroRNAs in plants. Genes Dev. 2002;16(13):1616-26.

4. Axtell MJ. Classification and Comparison of Small RNAs from Plants. Annu Rev Plant Biol 2013:64:137-59.

5. Mosher RA, Tan EH, Shin J, Fischer RL, Pikaard CS, Baulcombe DC. An atypical epigenetic mechanism affects uniparental expression of Pol IVdependent siRNAs. PLoS One. 2011;6(10), e25756.

6. Ratcliff F, Harrison BD, Baulcombe DC. A similarity between viral defense and gene silencing in plants. Science. 1997;276(5318):1558-60.

7. Zvereva AS, Pooggin MM. Silencing and innate immunity in plant defense against viral and non-viral pathogens. Viruses. 2012;4(11):2578-97.

8. Ito H. Small RNAs and transposon silencing in plants. Dev Growth Differ. 2012:54(1):100-7.

9. Castel SE, Martienssen RA. RNA interference in the nucleus: roles for small RNAs in transcription, epigenetics and beyond. Nat Rev Genet. 2013;14(2):100-12.

10. Lau NC, Lim LP, Weinstein EG, Bartel DP. An abundant class of tiny RNAs with probable regulatory roles in Caenorhabditis elegans. Science. 2001; 294(5543):858-62.

11. Lee RC, Feinbaum RL, Ambros $V$. The $C$. elegans heterochronic gene lin- 4 encodes small RNAs with antisense complementarity to lin-14. Cell. 1993;75(5):843-54.

12. Llave C, Xie Z, Kasschau KD, Carrington JC. Cleavage of Scarecrow-like mRNA targets directed by a class of Arabidopsis miRNA. Science. 2002; 297(5589):2053-6.

13. Bartel DP. MicroRNAs: genomics, biogenesis, mechanism, and function. Cell. 2004;116(2):281-97.

14. Baulcombe D. RNA silencing in plants. Nature. 2004;431(7006):356-63.

15. Voinnet O. Origin, biogenesis and activity of plant microRNAs. Cell. 2009; 136(4):669-87.

16. $\mathrm{Ko} \mathrm{JH}$, Prassinos $\mathrm{C}$, Han $\mathrm{KH}$. Developmental and seasonal expression of PtaHB1, a Populus gene encoding a class III HD-Zip protein, is closely associated with secondary growth and inversely correlated with the level of microRNA (miR166). New Phytol. 2006;169(3):469-78

17. Zhou M, Li D, Li Z, Hu Q, Yang C, Zhu L, Luo H. Constitutive expression of a miR319 gene alters plant development and enhances salt and drought tolerance in transgenic creeping bentgrass (Agrostis stolonifera L.). Plant Physiol 2013;161(3):1375-91.

18. Wang ZJ, Huang JQ, Huang YJ, Li Z, Zheng BS. Discovery and profiling of novel and conserved microRNAs during flower development in Carya cathayensis via deep sequencing. Planta. 2012;236(2):613-21.

19. Wu G, Poethig RS. Temporal regulation of shoot development in Arabidopsis thaliana by miR156 and its target SPL3. Development 2006:133(18):3539-47.

20. Yamaguchi A, Abe M. Regulation of reproductive development by non-coding RNA in Arabidopsis: to flower or not to flower. J Plant Res. 2012;125(6):693-704

21. Wang K, Li M, Gao F, Li S, Zhu Y, Yang P. Identification of conserved and novel microRNAs from Liriodendron chinense floral tissues. PLoS One. 2012;7(9), e44696.

22. Eckardt NA. A microRNA cascade in plant defense. Plant Cell. 2012:24(3):840.

23. Zhao JP, Jiang XL, Zhang BY, Su XH. Involvement of microRNA-mediated gene expression regulation in the pathological development of stem canker disease in Populus trichocarpa. PLoS One. 2012;7(9), e44968.

24. Staiger D, Korneli C, Lummer M, Navarro L. Emerging role for RNA-based regulation in plant immunity. New Phytol. 2012;197(2):394-404.

25. Zeng QY, Yang CY, Ma QB, Li XP, Dong WW, Nian H. Identification of wild soybean miRNAs and their target genes responsive to aluminum stress. BMC Plant Biol. 2012:12:182

26. Ferreira $\mathrm{TH}$, Gentile A, Vilela RD, Costa GG, Dias LI, Endres L, et al. microRNAs associated with drought response in the bioenergy crop sugarcane (Saccharum spp.). PLoS One. 2012;7(10):e46703.

27. Eldem V, Celikkol Akcay U, Ozhuner E, Bakir Y, Uranbey S, Unver T. Genomewide identification of miRNAs responsive to drought in peach (Prunus persica) by high-throughput deep sequencing. PLoS One. 2012;7(12), e50298.

28. Morin RD, Aksay G, Dolgosheina E, Ebhardt HA, Magrini V, Mardis ER, et al. Comparative analysis of the small RNA transcriptomes of Pinus contorta and Oryza sativa. Genome Res. 2008;18(4):571-4

29. Rajagopalan R, Vaucheret H, Trejo J, Bartel DP. A diverse and evolutionarily fluid set of microRNAs in Arabidopsis thaliana. Genes Dev. 2006:20:3407-25.

30. Yao Y, Guo G, Ni Z, Sunkar R, Du J, Zhu JK, et al. Cloning and characterization of microRNAs from wheat (Triticum aestivum L.). Genome Biol. 2007;8(6):R96. 
31. Moxon S, Jing R, Szittya G, Schwach F, Rusholme Pilcher RL, Moulton V, et al. Deep sequencing of tomato short RNAs identifies microRNAs targeting genes involved in fruit ripening. Genome Res. 2008;18(10):1602-9.

32. Zhu QH, Spriggs A, Matthew L, Fan L, Kennedy G, Gubler F, et al. A diverse set of microRNAs and microRNA-like small RNAs in developing rice grains. Genome Res. 2008;18(9):1456-65.

33. Hsieh LC, Lin SI, Shih AC, Chen JW, Lin WY, Tseng CY, et al. Uncovering small RNA-mediated responses to phosphate deficiency in Arabidopsis by deep sequencing. Plant Physiol. 2009;151(4):2120-32.

34. Guzman F, Almerao MP, Korbes AP, Loss-Morais G, Margis R. Identification of microRNAs from Eugenia uniflora by high-throughput sequencing and bioinformatics analysis. PLoS One. 2012;7(11), e49811.

35. Wan LC, Wang F, Guo X, Lu S, Qiu Z, Zhao Y, et al. Identification and characterization of small non-coding RNAs from Chinese fir by high throughput sequencing. BMC Plant Biol. 2012;12:146.

36. Zhang Y, Bai Y, Han J, Chen M, Kayesh E, Jiang W, et al. Bioinformatics prediction of miRNAs in the Prunus persica genome with validation of their precise sequences by miR-RACE. J Plant Physiol. 2012;170(1):80-92.

37. Meyers BC, Axtell MJ, Bartel B, Bartel DP, Baulcombe D, Bowman JL, et al, Criteria for annotation of plant MicroRNAs. Plant Cell. 2008;20(12):3186-90.

38. Axtell MJ, Snyder JA, Bartel DP. Common functions for diverse small RNAs of land plants. Plant Cell. 2007;19(6):1750-69.

39. Arazi T, Talmor-Neiman M, Stav R, Riese M, Huijser P, Baulcombe DC. Cloning and characterization of micro-RNAs from moss. Plant J. 2005;43(6):837-48.

40. Lu S, Sun YH, Shi R, Clark C, Li L, Chiang VL. Novel and mechanical stressresponsive MicroRNAs in Populus trichocarpa that are absent from Arabidopsis. Plant Cell. 2005;17(8):2186-203.

41. Talmor-Neiman M, Stav R, Frank W, Voss B, Arazi T. Novel micro-RNAs and intermediates of micro-RNA biogenesis from moss. Plant J. 2006;47(1):25-37

42. Heisel SE, Zhang Y, Allen E, Guo L, Reynolds TL, Yang X, et al. Characterization of unique small RNA populations from rice grain. PLoS One. 2008;3(8), e2871.

43. Kozomara A, Griffiths-Jones S. miRBase: integrating microRNA annotation and deep-sequencing data. Nucleic Acids Res. 2011;39(Database issue):D152-157.

44. Tuskan GA, Difazio S, Jansson S, Bohlmann J, Grigoriev I, Hellsten U, et al. The genome of black cottonwood, Populus trichocarpa (Torr. \& Gray). Science. 2006:313(5793):1596-604.

45. Xia R, Zhu H, An YQ, Beers EP, Liu Z. Apple miRNAs and tasiRNAs with novel regulatory networks. Genome Biol. 2012;13(6):R47.

46. Wan LC, Zhang H, Lu S, Zhang L, Qiu Z, Zhao Y, et al. Transcriptome-wide identification and characterization of miRNAs from Pinus densata. BMC Genomics. 2012:13:132

47. Gebelin V, Argout X, Engchuan W, Pitollat B, Duan C, Montoro P, et al. Identification of novel microRNAs in Hevea brasiliensis and computational prediction of their targets. BMC Plant Biol. 2012;12:18.

48. Zhu H, Xia R, Zhao B, An YQ, Dardick CD, Callahan AM, et al. Unique expression, processing regulation, and regulatory network of peach (Prunus persica) miRNAs. BMC Plant Biol. 2012;12:149.

49. Ladiges PY, Udovicic F, Nelson G. Australian biogeographical connections and the phylogeny of large genera in the plant family Myrtaceae. J Biogeogr. 2003;30(7):989-98.

50. Gaiotto FA, Bramucci M, Grattapaglia D. Estimation of outcrossing rate in a breeding population of Eucalyptus urophylla s.t. Blake with dominant RAPD and AFLP markers. Theor Appl Genet. 1997;95:842-9.

51. Moran GJCB, Griffin AR. Reduction in levels of inbreeding in a seed orchard of Eucalyptus regnans F. Muell, compared with natural populations. Silvae Genetica. 1989:38:32-6.

52. Grattapaglia D, Kirst M. Eucalyptus applied genomics: from gene sequences to breeding tools. New Phytol. 2008;179(4):911-29.

53. FAO. Global Wood And Wood Products Flow. In: FAO Forestry Paper. Shangai: FAO: FAO; 2007.

54. Myburg AA, Grattapaglia D, Tuskan GA, Hellsten U, Hayes RD, Grimwood J et al. The genome of Eucalyptus grandis. Nature 2014.

55. Goodstein DM, Shu S, Howson R, Neupane R, Hayes RD, Fazo J, et al. Phytozome: a comparative platform for green plant genomics. Nucleic Acids Res. 2011:40(Database issue):D1178-1186.

56. Li H, Durbin R. Fast and accurate short read alignment with BurrowsWheeler transform. Bioinformatics. 2009:25(14):1754-60.

57. Prufer K, Stenzel U, Dannemann M, Green RE, Lachmann M, Kelso J. PatMaN: rapid alignment of short sequences to large databases. Bioinformatics. 2008;24(13):1530-1.
58. Manavella PA, Koenig D, Weigel D. Plant secondary siRNA production determined by microRNA-duplex structure. Proc Natl Acad Sci U S A. 2012;109(7):2461-6.

59. Yoshikawa M, Peragine A, Park MY, Poethig RS. A pathway for the biogenesis of trans-acting siRNAs in Arabidopsis. Genes Dev. 2005;19(18):2164-75.

60. Friedlander MR, Mackowiak SD, Li N, Chen W, Rajewsky N. miRDeep2 accurately identifies known and hundreds of novel microRNA genes in seven animal clades. Nucleic Acids Res. 2011;40(1):37-52.

61. Dai X, Zhao PX. psRNATarget: a plant small RNA target analysis server. Nucleic Acids Res. 2011;39(Web Server issue):W155-159.

62. Crooks GE, Hon G, Chandonia JM, Brenner SE. WebLogo: a sequence logo generator. Genome Res. 2004;14(6):1188-90

63. Mao W, Li Z, Xia X, Li Y, YU J. A combined approach of high-throughput sequencing and degradome analysis reveals tissue specific expression of microRNAs and their targets in cucumber. PLoS One. 2012;7(3):e33040.

64. Shamimuzzaman M, Vodkin L. Identification of soybean seed developmental stage-specific and tissue-specific miRNA targets by degradome sequencing. BMC Genomics. 2012;13:310.

65. Rhoades MW, Reinhart BJ, Lim LP, Burge CB, Bartel B, Bartel DP. Prediction of plant microRNA targets. Cell. 2002;110(4):513-20.

66. Xie K, Wu C, Xiong L. Genomic organization, differential expression, and interaction of SQUAMOSA promoter-binding-like transcription factors and microRNA156 in rice. Plant Physiol. 2006;142(1):280-93.

67. Xing S, Salinas M, Hohmann S, Berndtgen R, Huijser P. miR156-targeted and nontargeted SBP-box transcription factors act in concert to secure male fertility in Arabidopsis. Plant Cell. 2010;22(12):3935-50.

68. Liu X, Huang J, Wang Y, Khanna K, Xie Z, Owen HA, et al. The role of floral organs in carpels, an Arabidopsis loss-of-function mutation in MicroRNA160a, in organogenesis and the mechanism regulating its expression. Plant J. 2010;62(3):416-28.

69. Mallory AC, Bartel DP, Bartel B. MicroRNA-directed regulation of Arabidopsis AUXIN RESPONSE FACTOR17 is essential for proper development and modulates expression of early auxin response genes. Plant Cell. 2005;17(5):1360-75.

70. Aukerman MJ, Sakai H. Regulation of flowering time and floral organ identity by a MicroRNA and its APETALA2-like target genes. Plant Cell. 2003:15(11):2730-41.

71. Zhu $\mathrm{QH}$, Helliwell CA. Regulation of flowering time and floral patterning by miR172. J Exp Bot. 2011;62(2):487-95.

72. Chen D, Meng Y, Ma X, Mao C, Bai Y, Cao J, et al. Small RNAs in angiosperms: sequence characteristics, distribution and generation. Bioinformatics. 2010;26(11):1391-4.

73. Ong SS, Wickneswari R. Expression profile of small RNAs in Acacia mangium secondary xylem tissue with contrasting lignin content - potential regulatory sequences in monolignol biosynthetic pathway. BMC Genomics. 2012;12 Suppl 3:S13.

74. Wu L, Zhou H, Zhang Q, Zhang J, Ni F, Liu C, et al. DNA methylation mediated by a microRNA pathway. Mol Cell. 2010;38(3):465-75.

75. Cuperus JT, Fahlgren N, Carrington JC. Evolution and functional diversification of MIRNA genes. Plant Cell. 2011;23(2):431-42.

76. Carthew RW, Sontheimer EJ. Origins and Mechanisms of miRNAs and siRNAs. Cell. 2009;136(4):642-55.

77. Zhai J, Jeong DH, De Paoli E, Park S, Rosen BD, Li Y, et al. MicroRNAs as master regulators of the plant NB-LRR defense gene family via the production of phased, trans-acting siRNAs. Genes Dev. 2011;25(23):2540-53.

78. Zhong $\mathrm{R}$, Richardson EA, Ye ZH. The MYB46 transcription factor is a direct target of SND1 and regulates secondary wall biosynthesis in Arabidopsis. Plant Cell. 2007;19(9):2776-92.

79. Bedon F, Grima-Pettenati J, Mackay J. Conifer R2R3-MYB transcription factors: sequence analyses and gene expression in wood-forming tissues of white spruce (Picea glauca). BMC Plant Biol. 2007;7:17.

80. Patzlaff A, McInnis S, Courtenay A, Surman C, Newman LJ, Smith C, et al. Characterisation of a pine MYB that regulates lignification. Plant J. 2003;36(6):743-54

81. Schuler MA, Werck-Reichhart D. Functional genomics of P450s. Annu Rev Plant Biol. 2003;54:629-67.

82. Wang JW, Park MY, Wang LJ, Koo Y, Chen XY, Weigel D, et al. miRNA control of vegetative phase change in trees. PLOS Genet. 2011:7(2):e1002012

83. Wu G, Park MY, Conway SR, Wang JW, Weigel D, Poethig RS. The sequential action of miR156 and miR172 regulates developmental timing in Arabidopsis. Cell. 2009;138(4):750-9. 
84. Levy A, Szwerdszarf D, Abu-Abied M, Mordehaev I, Yaniv Y, Riov J, et al. Profiling microRNAs in Eucalyptus grandis reveals no mutual relationship between alterations in miR156 and miR172 expression and adventitious root induction during development. BMC Genomics. 2014;15:524.

85. Chang S, Puryear J, Cairney J. A simple and efficient method for isolating RNA from pine trees. Plant Mol Biol Report. 1993;11(2):113-6.

86. Edgar RC. Search and clustering orders of magnitude faster than BLAST. Bioinformatics. 2010;26(19):2460-1.

87. Griffiths-Jones S, Saini HK, van Dongen S, Enright AJ. miRBase: tools for microRNA genomics. Nucleic Acids Res. 2008;36(Database issue):D154-158.

88. Addo-Quaye C, Miller W, Axtell MJ. CleaveLand: a pipeline for using degradome data to find cleaved small RNA targets. Bioinformatics. 2009;25(1):130-1.

Submit your next manuscript to BioMed Central and we will help you at every step:

- We accept pre-submission inquiries

- Our selector tool helps you to find the most relevant journal

- We provide round the clock customer support

- Convenient online submission

- Thorough peer review

- Inclusion in PubMed and all major indexing services

- Maximum visibility for your research

Submit your manuscript at www.biomedcentral.com/submit 